\title{
LMI-Based Model Predictive Control for Underactuated Surface Vessels with Input Constraints
}

\author{
Lutao Liu, ${ }^{1}$ Zhilin Liu, $^{2}$ and Jun Zhang $^{3}$ \\ ${ }^{1}$ College of Information and Telecommunication, Harbin Engineering University, Harbin, Heilongjiang 150001, China \\ ${ }^{2}$ College of Automation, Harbin Engineering University, Harbin, Heilongjiang 150001, China \\ ${ }^{3}$ School of Electrical and Information Engineering, Jiangsu University, Zhenjiang, Jiangsu 212013, China
}

Correspondence should be addressed to Zhilin Liu; liuzhilin@hrbeu.edu.cn

Received 23 April 2014; Revised 27 June 2014; Accepted 27 June 2014; Published 14 July 2014

Academic Editor: Hamid R. Karimi

Copyright (C) 2014 Lutao Liu et al. This is an open access article distributed under the Creative Commons Attribution License, which permits unrestricted use, distribution, and reproduction in any medium, provided the original work is properly cited.

\begin{abstract}
A nonlinear model predictive control (MPC) is proposed for underactuated surface vessel (USV) with constrained inputs. Aimed at the special structure of USV, a state-dependent coefficient (SDC) under the given USV is constructed in terms of diffeomorphism and state-dependent Riccati equation (SDRE) theory. Based on linear matrix inequalities (LMIs), the states of the USV are steered into an operating region around zero. When the states reach the region, the control law is switched to stabilize the system. And the constrained control input of the considered system is solved by convex optimization based on MPC involving LMIs. The simulation results verified the effectiveness of the proposed method. It is shown that, based on LMIs, it is easy to get the MPC for the USV with input constraints.
\end{abstract}

\section{Introduction}

Over the past decade, control of underactuated systems has been one of active research areas in control society. One feature of underactuated systems is that the number of independent actuators of the system is less than that of the degree of freedom. The challenging control problem is how to design a stabilizing control law such that the state of the closed-loop system asymptotically converges to the origin. In this note, we consider the dynamic positioningcontrol problem for a ship that has no side thruster, but two independent main thrusters located in a distance from the center line in order to provide both surge force and yaw moment. The control problem considered in this note is to find a feedback law that stabilizes both the position variables and the orientation, using only the two available controls. Since we attempt to control three degrees of freedom with only two independent controls, we have an underactuated control problem.

The dynamics of this underactuated system is complex enough to yield a rich source of control problems, yet simple enough to permit a complete mathematical analysis. It has been shown in [1] that underactuated vessels do not satisfy Brockett's necessary condition [2] if the unactuated dynamics contain no gravitational field component. In this case, these vessels are not asymptotically stabilizable to a given equilibrium configuration via time-invariant continuous feedback laws.

Stabilization of underactuated surface vessels has been tackled in a number of research studies in the last few years [3-6]. The controller in [7], which can be viewed as a time-varying controller devised to circumvent Brockett's limitation, is proposed. It consists of a supervising logic unit and subordinate time-invariant controllers. The asymptotic stability is rigorously proven using Lyapunov analysis. A sliding mode control (SMC) law [8] is presented by introducing a first-order sliding surface in terms of surge tracking errors and a second-order surface in terms of lateral motion tracking errors. In [9], a discontinuous feedback control law has been discussed for the exponential convergence of the equilibrium point under certain assumptions on the variables initial value. Contrastively, several transformations in [10] are introduced to represent the system into a nonlinear cascade form. One of the desirable features of the suggested 
approach in [10] is that it provides a systematic procedure to transform a class of nonholonomic dynamical systems into a nonlinear cascade form. Such representation opens the possibility of developing more constructive control tools than those already existing in [9]. However, it is a pity that the control design in [10] is not complete and lacked a further step in the backstepping procedure. In the comment letter [11], the control laws in [10] were revised and the states decayed asymptotically to zero. In [12], a discontinuous control approach with two stage control laws switched on at given time is proposed based on the stability analysis of the global transformed system. With the aid of terminal sliding mode (TSM) method, the two asymptotically stabilizing control laws for the transformed subsystems are designed independently to asymptotically stabilize the underactuated surface vessel. A controller designed by using Lyapunov direct method and the popular backstepping techniques [13] is utilized to stabilize the USV system to the origin.

In realistic implementations, the performance of control systems is often limited due to constraints on inputs or states that naturally arise. None of the references cited above has taken those constraints explicitly into account. MPC is a control technique which embeds optimization within feedback to deal with systems subject to constraints on inputs and states [14]. Over the last few decades, MPC has proven to be successful for a wide range of applications including chemical, food processing, automotive, and aerospace systems [15]. The fact that the rudder actuation is limited in amplitude and rate makes MPC approach a natural choice for the design of the path following controller. By taking these physical constraints into account, control actions that respect actuators limits can be generated. As the pioneer work of MPC application in control of surface vessels, authors in [16] consider rudder saturation in their MPC controller and adopt a 1DoF yaw dynamical model. By using model predictive control algorithm, a rudder roll stabilization (RRS) system is presented in [17]. The control objective is to regulate the heading to a desired value and to reduce the roll angle under various sea conditions. In [18], a nonlinear MPC control law with terminal invariant manifolds constraints is designed through coordinate transformation and state feedback transformation based on diffeomorphism and Lyapunov stability theory. Based on MPC, the states of the USV are steered into a terminal manifolds set. After the terminal manifolds set is reached, a linear feedback control is used to stabilize the system. However, just as the authors said in their paper, the control design proposed is developed with constant parameters without considering parameter perturbation. In [19], the focus is on satisfying all the input (rudder) and state (roll) constraints by using MPC to achieve satisfactory path following performance. Authors in [20] present an experimental implementation of a model predictive control (MPC) strategy for path following on a model ship. The objective of the proposed algorithm is to control cross-tracking error and heading angle using both linearized and nonlinear models. To implement MPC with a nonlinear model, the integrated perturbation analysis and sequential quadratic programming (InPA-SQP) method are introduced to solve the constrained optimal control problem.



FIGURE 1: Underactuated surface vessel.

For notational convenience, the ship dynamics in $[19,20]$ are written into linearized and nonlinear form based on the assumption that surge velocity is constant and the yaw moment is proportional to the rudder angle. However, the former assumption is hardly admissible in engineering.

This paper illuminates the stabilization approach for underactuated surface vessels with only a surge force and a yaw moment. The technique in this paper leads to convex LMIs based online optimization problem when the USV model is described by SDC in terms of diffeomorphism and SDRE theory. Moreover, the constrained control input, index, and stability of the considered system are solved by convex optimization based on LMIs. The simulation results involving an actual underactuated ship Northern Clipper [21] verified the effectiveness of the proposed method.

Notation. The symbol $*$ will be used in some matrix expressions to induce a symmetric structure. $I$ denotes identity matrix. For example, when $H$ and $R$ are symmetric matrices, then

$$
\left[\begin{array}{cc}
H & * \\
T & R
\end{array}\right]=\left[\begin{array}{cc}
H & T^{T} \\
T & R
\end{array}\right]
$$

\section{Problem Formulation}

In this paper, we consider the trajectory tracking control problem of a surface vessel shown in Figure 1. There is no side thruster, but two independent main thrusters located in a distance from the center line in order to provide both surge force and yaw moment.

The dynamics and kinematics of an underactuated surface vessel are described as [22]

$$
\begin{gathered}
M \dot{\nu}+C(\nu) \nu+D \nu=\tau \\
\dot{\eta}=J(\eta) \nu .
\end{gathered}
$$

The inertia matrix $M=\operatorname{diag}\left\{m_{11}, m_{22}, m_{33}\right\}$ and the damping matrix $D=\operatorname{diag}\left\{d_{11}, d_{22}, d_{33}\right\}$ are constant and positive definite. The vector $\tau=\left[\tau_{1}, \tau_{2}, \tau_{3}\right]$ denotes the control forces in surge and sway and control torque in yaw. In this paper, the surface vessel is considered as the common thruster configuration that has no side thruster, such as $\tau_{2}=$ 
0 . So, the second component equation of (2) behaves as a nonholonomic constraint, which is a nonintegrable relation involving not only the generalized coordinates and velocities but also the generalized accelerations [23]. $C(\nu)$ is the matrix of Coriolis and centripetal terms also including added mass. $J(\eta)$ is the rotation matrix for the transformation between body-fixed and earth-fixed coordinates. Consider

$$
\begin{aligned}
& C(\nu)=\left[\begin{array}{ccc}
0 & 0 & -m_{22} v \\
0 & 0 & m_{11} u \\
m_{22} v & -m_{11} u & 0
\end{array}\right] \\
& J(\eta)=\left[\begin{array}{ccc}
\cos (\psi) & -\sin (\psi) & 0 \\
\sin (\psi) & \cos (\psi) & 0 \\
0 & 0 & 1
\end{array}\right] .
\end{aligned}
$$

The vector $\eta=[x, y, \psi]^{T}$ denotes the positions and orientation of the underactuated surface vessel in the earth-fixed coordinate system. The vector $v=[u, v, r]^{T}$ denotes the linear velocities in surge and sway and the angular velocity in yaw.

As a general accepted conclusion (see $[24,25]$ ), there is no continuous time-invariant feedback control law that makes the zero origin an asymptotically stable equilibrium of the system (2)-(3), for the system does not satisfy Brockett's condition [2]. Then time-varying and discontinuous control approaches are only taken into account in this paper.

Neglecting the motions in heave, roll, and pitch, the simplified kinematic model which describes the geometrical relationship between the earth-fixed (E-frame) and the bodyfixed (B-frame) motion is given as

$$
\begin{gathered}
m_{11} \dot{u}-m_{22} v r+d_{11} u=\tau_{1} \\
m_{22} \dot{v}+m_{11} u r+d_{22} v=0 \\
m_{33} \dot{r}+\left(m_{22}-m_{11}\right) u v+d_{33} r=\tau_{3} \\
\dot{x}=u \cos \psi-v \sin \psi \\
\dot{y}=u \sin \psi+v \cos \psi \\
\dot{\psi}=r .
\end{gathered}
$$

The following global coordinate transformation and feedback transformation are adopted before control design. Define

$$
\begin{aligned}
& z_{1}=x \cos (\psi)+y \sin (\psi) \\
& z_{2}=v \\
& z_{3}=-x \sin (\psi)+y \cos (\psi)+\frac{m_{22}}{d_{22} v} \\
& z_{4}=\psi \\
& z_{5}=-\frac{m_{11}}{d_{22} u}-z_{1} \\
& z_{6}=r .
\end{aligned}
$$

It is proved that the state transformation (6) is a global diffeomorphism (see [22]).
The feedback transformation is

$$
\begin{aligned}
& w_{1}=\left(\frac{d_{11}}{d_{22}}-1\right) u-z_{3} z_{6}-\frac{\tau_{1}}{d_{22}} \\
& w_{2}=\frac{\left(m_{11}-m_{22}\right) u v}{m_{33}}-\frac{d_{33} r}{m_{33}}+\frac{\tau_{3}}{m_{33}} .
\end{aligned}
$$

With the state and feedback transformation (6)-(7), the system (2)-(3) is eventually transformed to

$$
\begin{aligned}
& \dot{z}_{1}=-\frac{d_{22}}{m_{11}} z_{1}-\frac{d_{22}}{m_{11}} z_{5}+z_{3} z_{6}-\frac{m_{22}}{d_{22}} z_{2} z_{6} \\
& \dot{z}_{2}=-\frac{d_{22}}{m_{22}} z_{2}+\frac{d_{22}}{m_{22}} z_{6}\left(z_{1}+z_{5}\right) \\
& \dot{z}_{3}=z_{5} z_{6} \\
& \dot{z}_{4}=z_{6} \\
& \dot{z}_{5}=w_{1} \\
& \dot{z}_{6}=w_{2} .
\end{aligned}
$$

The system (8) has the same diffeomorphism properties as the system (2)-(3) (see [22]); that is, if $\lim _{t \rightarrow \infty} z_{i}=0,(1 \leq i \leq 6)$ then $(x, y, \psi, u, v, r)$ converges to zero as $t \rightarrow \infty$.

\section{Control Design}

MPC is a popular technique for the control of dynamical system subject to input and state constraints. At any time instant, MPC requires the online solution of an optimization problem to compute optimal control inputs over a fixed number of future time instants, known as the finite horizon or quasi-infinite horizon. Using MPC, it is possible to handle inequality constraints on the manipulated and controlled variables in a systematic manner during the design and implementation of the controller (see [26]).

We will consider a nonlinear plant with input constraints, where the evolution of the state after time is predicted by the following model:

$$
\begin{array}{ll}
\min _{u(\cdot)} & J(x, t, u) \\
\text { s.t. } & \dot{x}(\tau)=f(x(\tau), u(\tau)), \quad \tau \in\left[t, t+T_{p}\right], x(0)=x_{0} \\
& u(\tau) \in U, \quad \tau \in\left[t, t+T_{p}\right] \\
& x(\tau) \in X, \quad \tau \in\left[t, t+T_{p}\right] \\
& x\left(t+T_{p}\right) \in W .
\end{array}
$$

The data of this model comprise a set $W \in R^{n}$ containing all possible terminal states with constraints, a vector $X$ that is the set of the state constraints, and a given vector $U$ of possible set of control constraints. Our objective is to obtain a feedback law that (asymptotically) drives the state of our 
plant to the origin. In this paper, we consider the following quadratic objective:

$$
J(x, t, u)=\int_{t}^{t+T_{p}} l(x(\tau), u(\tau)) d \tau+F\left(x\left(t+T_{p}\right)\right) .
$$

The domain of this optimization problem is the set of admissible processes, namely, pairs $(x, u)$, comprising a measurable control function $u$ and the corresponding absolutely continuous state trajectory $x$ which satisfy the constraints of $\min J(x, t, u)$, where function $F(\cdot) \geq 0$ is selected as terminal costs. We show that we can guarantee stability of the resultant closed-loop system, by choosing the design terminal constraints set $W$ and terminal costs $F(\cdot)$ to satisfy a certain stability condition. However, in the above technique, selecting nonlinear affine system (8) as the predictive model may give rise to a lot of problems such as complicated computation and difficulty solving the optimization; the problems inherent to nonlinear affine model are inevitable. In this work, we will make efforts to design MPC controllers involving SDC model to overcome the previous problem (see [27]). System (8) is equivalent to the following nonlinear affine model:

$$
\dot{x}=f(x)+g(x) u .
$$

It is well-known that the nonlinear dynamics (8) can be represented by the following linear structure having SDC (state-dependent coefficients) by using SDRE theory (see [28]):

$$
\dot{x}=A(x) x+B(x) u,
$$

where $f(x)=x A(x)$ and $B(x)=g(x)$. It is also known [28] that, in the multivariable case, there are an infinite number of ways to bring the nonlinear system to SDC form. It is clear that the modeling errors caused by the traditional linear methods are avoided.

Definition 1. $A(x)$ is a controllable (stabilizable) parameterization of the nonlinear system (in a region $X$ ) if the pair $(A(x), B(x))$ is pointwise controllable (stabilizable) in the linear sense for all $x \in X$ (see [28]).

Construct the nonlinear feedback controller via

$$
u(x)=-K(x) x=-R^{-1} B^{T}(x) P(x) x .
$$

Solve the state-dependent Riccati equation (SDRE)

$$
\begin{aligned}
& A^{T}(x) P(x)+P(x) A(x)+Q(x) \\
& \quad-P(x) B(x) R^{-1}(x) B^{T}(x) P(x)=0
\end{aligned}
$$

to obtain $P(x) \geq 0$. Note that $P(x)$ is a solution of $x$.

Physical limitations inherent in ship power transmission system invariably impose hard constraints on the manipulated variable input. In this section, we show how limits on the control signal can be incorporated into our MPC algorithm as sufficient LMI constraints. Predictive control is an effective optimization control method to deal with constrains. The basic idea of the discussion that follows can be found in [26] in the context of discrete-time robust MPC. We present it here to clarify its application in our underactuated surface vessels setting and also for completeness of exposition.

The nonlinear dynamics (8) can be represented by the following linear structure having state-dependent coefficients:

$$
\dot{z}=A(z) z+B(z) w
$$

where

$$
\begin{aligned}
& A(z)=\left[\begin{array}{cccccc}
-\frac{d_{22}}{m_{11}} & -\frac{m_{22}}{m_{11}} z_{6} & z_{6} & 0 & -\frac{d_{22}}{m_{11}} & 0 \\
\frac{d_{22}}{m_{22}} z_{6} & -\frac{d_{22}}{m_{22}} & 0 & 0 & \frac{d_{22}}{m_{22}} z_{6} & 0 \\
0 & 0 & 0 & 0 & \frac{z_{6}}{2} & \frac{z_{5}}{2} \\
0 & 0 & 0 & 0 & 0 & 1 \\
0 & 0 & 0 & 0 & 0 & 0 \\
0 & 0 & 0 & 0 & 0 & 0
\end{array}\right] \\
& B(z)=\left[\begin{array}{llllll}
0 & 0 & 0 & 0 & 1 & 0 \\
0 & 0 & 0 & 0 & 0 & 1
\end{array}\right]^{T}
\end{aligned}
$$

The Euler discrete system of (15) is given by

$$
\begin{aligned}
z(k+1) & =A(z, k) z(k)+B(z, k) w(k) \\
\|w\|_{2} & \leq w_{\max } .
\end{aligned}
$$

We define the nominal cost as

$$
\begin{aligned}
J_{\infty}= & \sum_{i=0}^{\infty} z^{T}(k+i \mid k) Q z(k+i \mid k) \\
& +w^{T}(k+i \mid k) R w(k+i \mid k),
\end{aligned}
$$

where $Q=Q^{T}>0$ and $R=R^{T}>0$. MPC is an attractive strategy for systems subject to input constraints. Due to the systems with constrained control input, we obtain the control input by minimizing a nominal cost $J_{\infty}$ over an infinite predictive horizon as follows:

$$
\min _{w(k+i \mid k)} J_{\infty}
$$

It is very difficult to resolve the optimization (20) directly. We can see that, based on LMI constraints, it is easy to get the optimization in system (20).

Theorem 2. Consider the time-varying system (18)-(20), where $z(k \mid k)$ is the state of the system (15) measured at sampling time $k . w_{\max }$ is the maximum value of the input $w$. When $A(x)$ is a controllable parameterization of the system (18), if there exist $F, Q, \gamma, Q=Q^{T} \in R^{6 \times 6}>0, \gamma \in R^{1 \times 1}>0$, and $Y \in R^{2 \times 6}$, the state feedback matrix $F$ in the control law $w=F z(k)$ that minimizes the upper bound on the performance objective function at sampling time $k$ is given by $F=Y Q^{-1} z(k)$, where $Q$ and $Y$ are obtained from the solution (if it exists) of the following linear objective minimization problem. If the 
optimization problem in (21)-(23) is feasible, the closed-loop underactuated system (11) with input constraints $\|w\|_{2} \leq w_{\max }$ is asymptotically stable. Consider

$$
\begin{aligned}
\min _{Y, Q, \gamma} & \gamma \\
\text { s.t. } & {\left[\begin{array}{cc}
1 & z^{T}(k) \\
z(k) & Q
\end{array}\right] \geq 0 } \\
& {\left[\begin{array}{cccc}
A(z, k) Q+B(z, k) Y & Q & 0 & 0 \\
Q_{1}^{1 / 2} Q & 0 & \gamma I & 0 \\
R^{1 / 2} Y & 0 & 0 & \gamma I
\end{array}\right]>0 } \\
& {\left[\begin{array}{ccc}
w_{\max }^{2} I & Y \\
Y^{T} & Q
\end{array}\right] \geq 0 . }
\end{aligned}
$$

Proof. Consider the following.

(1) Proof for Optimization in Performance Index. Using quadratic Lyapunov-Krasovskii function, the upper bound on the objective function $J_{\infty}$ is given as

$$
V(z(k))=z^{T}(k) P(k) z(k) P(k)>0 .
$$

To guarantee the existence of the upper bound on the performance index, underlying inequalities must be given as

$$
\begin{gathered}
V(z(k+i+1 \mid k))-V(z(k+i \mid k)) \\
<-\left(z^{T}(k+i \mid k) Q z(k+i \mid k)\right. \\
\left.\quad+w^{T}(k+i \mid k) R w(k+i \mid k)\right)
\end{gathered}
$$

with the conditions $V(x(\infty \mid k))=0$ and $x(\infty \mid k)=0$. Summing (22) from 0 to $\infty$ gives

$$
\begin{aligned}
J= & \sum_{i=0}^{\infty} z^{T}(k+i \mid k) Q z(k+i \mid k) \\
& +w^{T}(k+i \mid k) R w(k+i \mid k) \\
\leq & V(z(k \mid k)) \leq \gamma,
\end{aligned}
$$

where $Q=\gamma P^{-1}>0$.

Using the Schur-complement [29], we get (21).

(2) Proof for the Stability in System (18)-(19). Substituting (18) to (19) gives

$$
\begin{gathered}
z^{T}(k)\left[P-(A+B F)^{T} P(k)(A+B F)\right. \\
\left.-Q_{1}-F^{T} R F\right] z(k)>0
\end{gathered}
$$

where $w=F z(k)=Y Q^{-1} z(k)$; we get

$$
P-(A+B F)^{T} P(k)(A+B F)-Q_{1}-F^{T} R F>0 .
$$

Using the Schur-complement, we get

$$
\left[\begin{array}{cccc}
P & * & * & * \\
A(z, k) P+B(z, k) F & P & 0 & 0 \\
Q_{1}^{1 / 2} P & 0 & I & 0 \\
R^{1 / 2} F & 0 & 0 & I
\end{array}\right]>0 .
$$

Substituting $F=Y Q^{-1}$ and pre- and postmultiplying by $Q$ give LMI (22).

(3) Proof for Input Constraints. Consider the Euclidean norm constraint $\|w\|_{2} \leq w_{\max }$. The constraint is imposed on the present and the entire horizon of future manipulated variables. Following [26], we have that

$$
\begin{aligned}
V(z(k+i \mid k)) & =z^{T}(k+i \mid k) Q^{-1} z(k+i \mid k) \\
& \leq z^{T}(k \mid k) Q^{-1} z(k \mid k) \leq 1
\end{aligned}
$$

is state-invariant ellipsoid. So, we get

$$
\begin{aligned}
\max _{i \geq 0}\|w(i \mid k)\|^{2} & =\max _{i \geq 0}\left\|Y Q^{-1} z(i \mid k)\right\|^{2} \\
& \leq \lambda_{\max }\left(Q^{-1 / 2} Y^{T} Y Q^{-1 / 2}\right) \leq w_{\text {max }}^{2}
\end{aligned}
$$

Using the Schur-complement, we get

$$
\left[\begin{array}{cc}
w_{\max }^{2} I & Y \\
Y^{T} & Q
\end{array}\right] \geq 0
$$

Remark 3. The applicable conditions of Theorem 2 is that $A(x)$ is a controllable parameterization of the system (18). This means that the condition $z_{6} \neq 0$ must be satisfied. When $z_{6}=0$, we have

$$
A(z)=\left[\begin{array}{cccccc}
-\frac{d_{22}}{m_{11}} & 0 & 0 & 0 & -\frac{d_{22}}{m_{11}} & 0 \\
0 & -\frac{d_{22}}{m_{22}} & 0 & 0 & 0 & 0 \\
0 & 0 & 0 & 0 & 0 & \frac{z_{5}}{2} \\
0 & 0 & 0 & 0 & 0 & 1 \\
0 & 0 & 0 & 0 & 0 & 0 \\
0 & 0 & 0 & 0 & 0 & 0
\end{array}\right],
$$

where the pair $(A(x), B(x))$ is not pointwise controllable. To solve the prevous problem, we define a constant $z_{6}^{*}$ which is close to zero but not equal to zero. When $z_{6}$ is close to zero, 
let $z_{6}=z_{6}^{*}$; the time-varying state matrix $A(z)$ is substituted as

$$
A\left(z_{5}, k\right)=\left[\begin{array}{cccccc}
-\frac{d_{22}}{m_{11}} & -\frac{m_{22}}{m_{11}} z_{6}^{*} & z_{6}^{*} & 0 & -\frac{d_{22}}{m_{11}} & 0 \\
\frac{d_{22}}{m_{22}} z_{6}^{*} & -\frac{d_{22}}{m_{22}} & 0 & 0 & \frac{d_{22}}{m_{22}} z_{6}^{*} & 0 \\
0 & 0 & 0 & 0 & \frac{z_{6}^{*}}{2} & \frac{z_{5}}{2} \\
0 & 0 & 0 & 0 & 0 & 1 \\
0 & 0 & 0 & 0 & 0 & 0 \\
0 & 0 & 0 & 0 & 0 & 0
\end{array}\right] .
$$

Substituting (34) to (22) gives

$$
\left[\begin{array}{cccc}
Q & * & * & * \\
A\left(z_{5}, k\right) Q+B\left(z_{5}, k\right) Y & Q & 0 & 0 \\
Q_{1}^{1 / 2} Q & 0 & \gamma I & 0 \\
R^{1 / 2} Y & 0 & 0 & \gamma I
\end{array}\right]>0 .
$$

So, we get the following Theorem 4 .

Theorem 4. Consider the time-varying system (18)-(20), where $z(k \mid k)$ is the state of the system (15) measured at sampling time $k . w_{\max }$ is the maximum value of the input $w$. If there exist $F, Q, \gamma, Q=Q^{T} \in R^{6 \times 6}>0, \gamma \in R^{1 \times 1}>0$, and $Y \in R^{2 \times 6}$, the state feedback matrix $F$ in the control law $w=F z(k)$ that minimizes the upper bound on the performance objective function at sampling time $k$ is given by $F=Y Q^{-1} z(k)$, where $Q$ and $Y$ are obtained from the solution of the following linear objective minimization problem. Simultaneously, input constraints $\|w\|_{2} \leq w_{\max }$ are satisfied.

$$
\begin{array}{lll}
\min _{Y, Q, \gamma} & \gamma \\
\text { s.t. } & (21),(22),(23) & \text { if }\left|z_{6}\right| \geq z_{6}^{*} \\
& (21),(23),(35) \quad \text { if }\left|z_{6}\right|<z_{6}^{*} .
\end{array}
$$

Remark 5. After obtaining the MPC controller $w$, we can get the control input for the origin system (5) by inverse diffeomorphism

$$
\begin{aligned}
& \tau_{1}=\left(d_{11}-d_{22}\right) u-d_{22} z_{3} z_{6}-d_{22} w_{1} \\
& \tau_{3}=m_{33} w_{2}-\left(m_{11}-m_{22}\right) u v+d_{33} r .
\end{aligned}
$$

\section{Simulation Results}

In [21], an underactuated actual ship named "Northern Clipper" is introduced. Consider the simulation model with parameters as Northern Clipper in [21]: $m_{11}=5.312 \times 10^{6} \mathrm{~kg}$, $m_{22}=8.283 \times 10^{6} \mathrm{~kg}, m_{33}=3.745 \times 10^{6} \mathrm{~kg}, d_{11}=5.024 \times$ $10^{4} \mathrm{~kg} / \mathrm{s}, d_{22}=2.722 \times 10^{5} \mathrm{~kg} / \mathrm{s}$, and $d_{33}=1.189 \times 10^{8} \mathrm{~kg} / \mathrm{s}$. Length of "Northern Clipper" $L=76.2 \mathrm{~m}$, and mass $m=$ $4.6 \times 10^{6} \mathrm{~kg}$. This suggests that the system matrices should

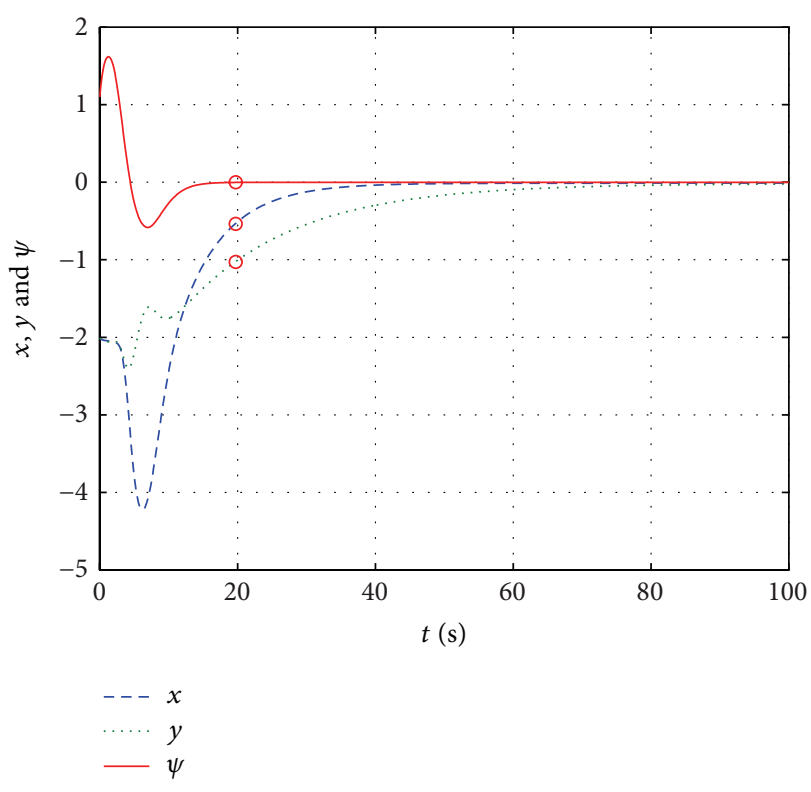

Figure 2: Response of $x, y$, and $\psi$.

be scaled with respect to forward speed, ship length $L$, and displacement (see [21]). The model parameters can be made nondimensional as $m_{11}^{\prime \prime}=1.1274, m_{22}^{\prime \prime}=1.8902, m_{33}^{\prime \prime}=$ $0.1278, d_{11}^{\prime \prime}=0.0358, d_{22}^{\prime \prime}=0.1183$, and $d_{33}^{\prime \prime}=0.0308$. In this section, the effectiveness of the proposed MPC control law is verified by following simulation. The control law is selected as Theorem 4 , and the initial values are selected as $x(0)=-2$, $y(0)=-2, \psi(0)=1, u(0)=v(0)=0 \mathrm{~m} / \mathrm{s}, r(0)=1, w_{\max }=1$, $z_{6}^{*}=0.001$, simulation time $T=100 \mathrm{~s}$, and sampling time $0.05 \mathrm{~s}$.

Simulation results are shown in the following figures, and the simulation time is set to $100 \mathrm{~s}$. Figure 2 gives the time response of the positions $x, y$ and orientation $\psi$. Figure 3 gives the time response of the velocities $u, v$ and orientation velocity $r$; Figure 4 gives the time response of $w_{1}, w_{2}$. The responses of the control inputs $\tau_{1}$ and $\tau_{3}$ are shown in Figure 5. The trajectory is shown in Figure 6. The switching motion of the control signal has been highlighted as red "o" on the simulation Figures $2-5$. Because $z_{6}^{*}$ is close to zero, the switching procedure is relatively smooth. Figures 2-6 show that the MPC control laws asymptotically stabilize the underactuated surface vessel to the zero origin. Moreover, the constraint on the control input is satisfied.

\section{Conclusion}

This paper proposes the stabilization approach for underactuated surface vessels with only a surge force and a yaw moment. The stability theory of MPC controller is further developed, which is applied to the stabilization control of underactuated surface vessel with input constraints. For the stabilization control of underactuated surface vessel, a nonlinear MPC control law is designed through coordinate transformation and SDRE based on LMIs. The simulation results show that 


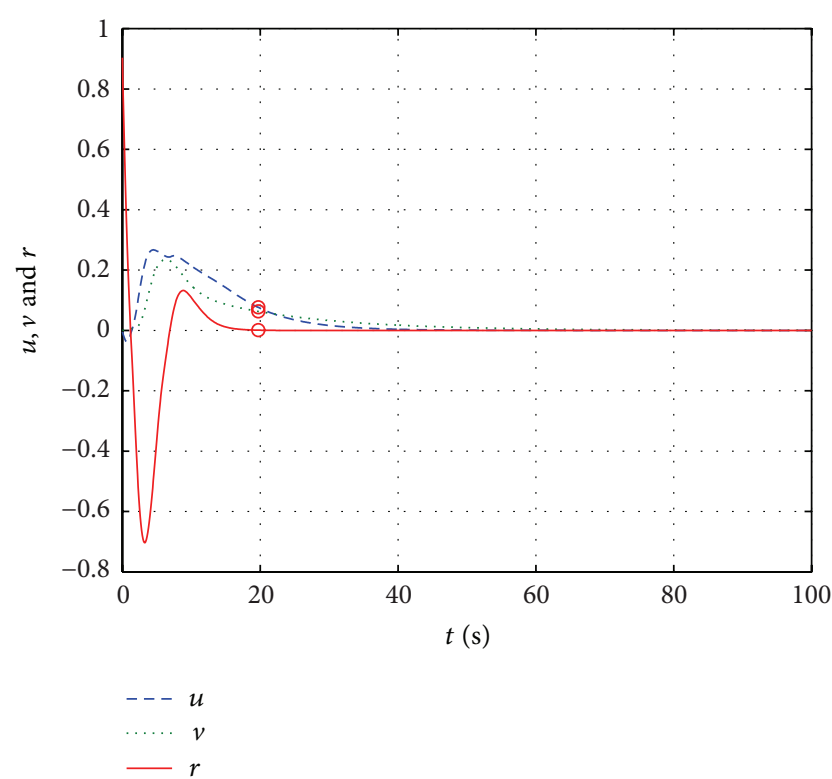

FIgURE 3: Response of $u, v$, and $r$.

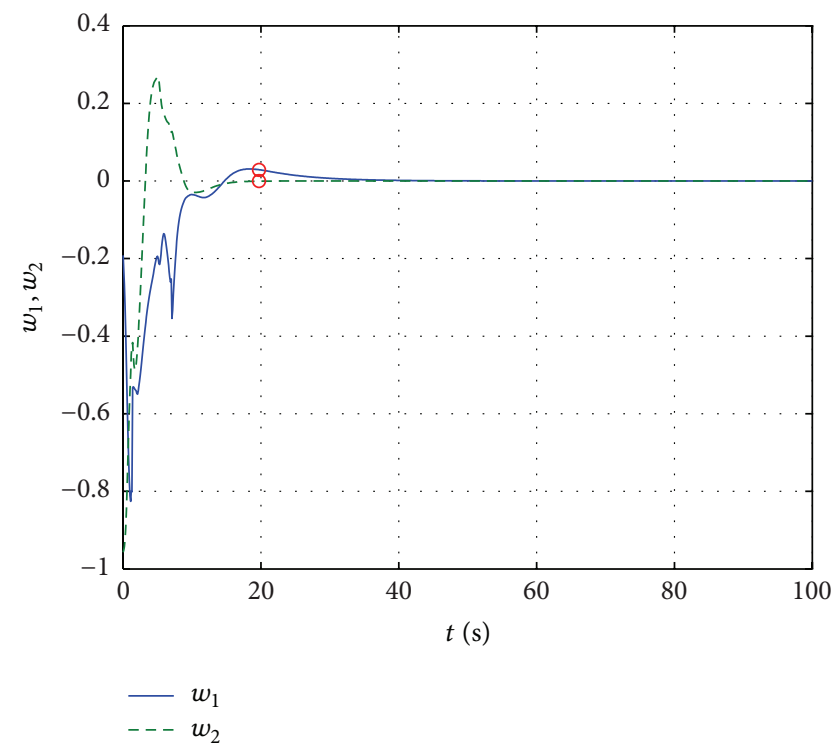

FIGURE 4: Response of $w_{1}, w_{2}$.

the proposed control law can effectively deal with the problem of stabilization control of underactuated surface vessel.

It should be noted that solving LMI itself is a challenge in computation. Many research efforts in LPV community are directed to address the computational issues. For LPV, those large LMIs are only solved once. But for MPC, the LMIs have to be solved repeatedly at each sampling time. In the future work, efforts will be made to analyze that whether the algorithms are fast enough to do this at the rate required by the control sampling. As is well known, for a long time, efficient setting of the large set of tunable parameters has been a hard problem for MPC. Many available methods to deal with uncertain systems have already been

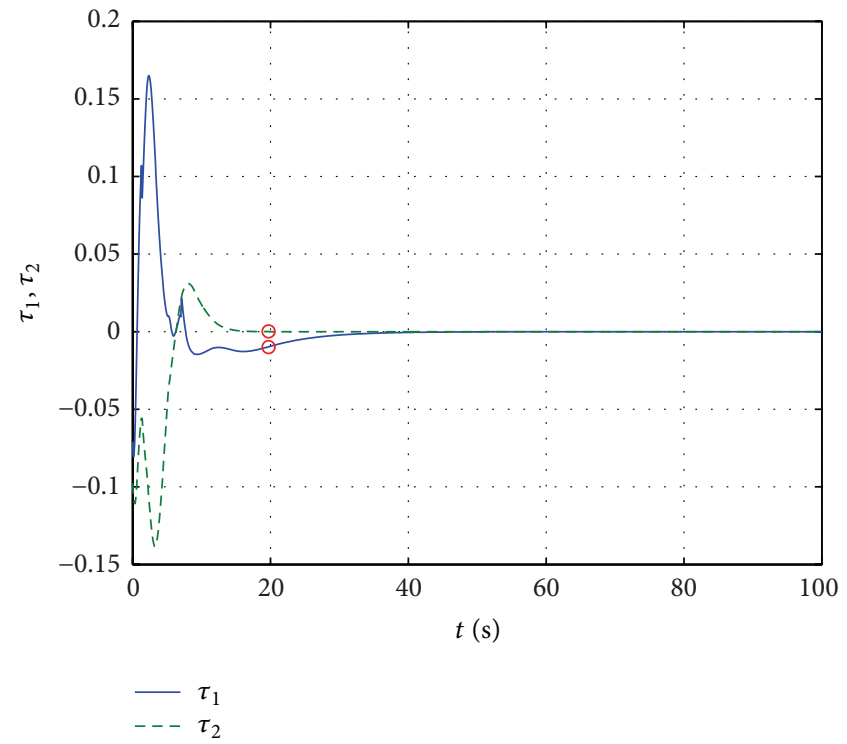

FIGURE 5: Control input.

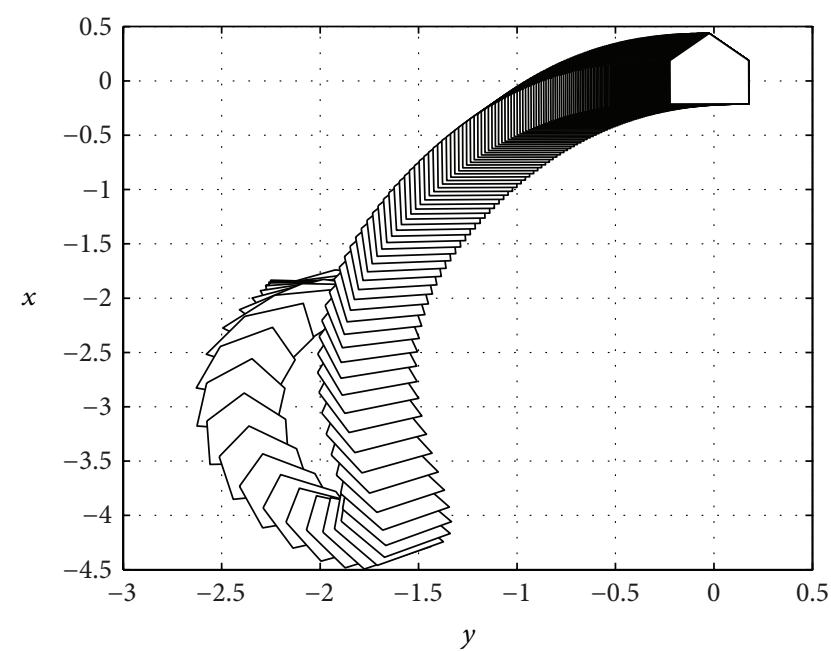

FIGURE 6: Trace of an underactuated surface vessel.

developed (see [30-33] and the references therein for more details). However, it should be noticed that MPC algorithm may perform very poorly when model mismatch occurs in spite of the inherent robustness provided by the feedback strategy based on the plant measurement at the next sampling time. MPC involving data-driven technique is suitable to overcome the previous problem [34-38]. In this paper, the robustness of the proposed approach was not discussed in detail. Fortunately, MPC requires the online solution of an optimization problem to compute optimal control inputs over a fixed number of future time instants, which partly guarantee the robustness of the dynamics. For the perturbed impulsive USV system with uncertainties, it is the further work that will solve the robust control problem in future. 


\section{Conflict of Interests}

The authors declare that there is no conflict of interests regarding the publication of this paper.

\section{Acknowledgments}

This work is partially supported by National Natural Science Foundation of China (61201410, 51379044), Fundamental Research Funds for the Central Universities (HEUCFX41304).

\section{References}

[1] K. Y. Wichlund, Q. J. Sordalen, and O. Egeland, "Control of vehicles with second holonomic constraints: underactuated vehicles," in Proceedings of the European Control Conference, pp. 3086-3091, 1995.

[2] R. W. Brockett, "Asymptotic stability and feedback stabilization," in Proceedings of Conference Progress in Mathematics, vol. 27, pp. 181-208, 1983.

[3] I. Fantoni, R. Lozano, F. Mazenc, and K. Y. Pettersen, "Stabilization of a nonlinear underactuated hovercraft," International Journal of Robust and Nonlinear Control, vol. 10, no. 8, pp. 645654, 2000.

[4] F. Mazenc, K. Y. Pettersen, and H. Nijmeijer, "Global uniform asymptotic stabilization of an underactuated surface vessel," in Proceedings of the 41st IEEE Conference on Decision and Control, pp. 510-515, December 2002.

[5] K. Y. Pettersen and H. Nijmeijer, "Global practical stabilization and tracking for an underactuated ship: a combined averaging and backstepping approach," in Proceedings of the IFAC Conference on Systems Structure and Control (SSC '98), pp. 59-64, Nantes, France, July 1998.

[6] K. Y. Pettersen and O. Egeland, "Exponential stabilization of an underactuated surface vessel," in Proceedings of the 35th IEEE Conference on Decision and Control, vol. 1, pp. 967-972, Kobe, Japan, December 1996.

[7] T.-H. Kim and T. Başar, "Asymptotic stabilization of an underactuated surface vessel via logic-based control," in Proceedings of the American Control Conference, vol. 6, pp. 4678-4683, May 2002.

[8] R. Yu, Q. Zhu, G. Xia, and Z. Liu, "Sliding mode tracking control of an underactuated surface vessel," IET Control Theory \& Applications, vol. 6, no. 3, pp. 461-466, 2012.

[9] M. Reyhanoglu, "Exponential stabilization of an underactuated autonomous surface vessel," Automatica, vol. 33, no. 12, pp. 2249-2254, 1997.

[10] J. Ghommam, F. Mnif, A. Benali, and N. Derbel, "Asymptotic backstepping stabilization of an underactuated surface vessel," IEEE Transactions on Control Systems Technology, vol. 14, no. 6, pp. 1150-1157, 2006.

[11] Z. Liu, R. Yu, and Q. Zhu, "Comments on asymptotic backstepping stabilization of an underactuated surface vessel," IEEE Transactions on Control Systems Technology, vol. 20, no. 1, pp. 286-288, 2012.

[12] J. Cheng, J. Yi, and D. Zhao, "Stabilization of an underactuated surface vessel via discontinuous control," in Proceedings of the American Control Conference, pp. 206-211, July 2007.

[13] G. Jawhar, M. Faical, and K. A. El-Metwally, "Backstepping technique for tracking control of an underactuated surface vessel with unmeasured thrust," in Proceedings of the IFAC, vol. 17, 2008.

[14] D. Q. Mayne, J. B. Rawlings, C. V. Rao, and P. O. M. Scokaert, "Constrained model predictive control: stability and optimality," Automatica, vol. 36, no. 6, pp. 789-814, 2000.

[15] S. J. Qin and T. A. Badgwell, "A survey of industrial model predictive control technology," Control Engineering Practice, vol. 11, no. 7, pp. 733-764, 2003.

[16] A. Wahl and E. D. Gilles, "Track-keeping on waterways using model predictive control," in Proceedings of the Workshop on Control Applications in Marine Systems, pp. 149-154, Elsevier, Oxford, UK, 1998.

[17] T. Perez, Ship Motion Control: Course Keeping and Roll Stabilisation Using Rudder and Fins, Springer, Berlin, Germany, 2005.

[18] L. Liu, Z. Liu, and J. Zhang, "Nonlinear model predictive control with terminal invariant manifolds for stabilization of underactuated surface vessel," Abstract and Applied Analysis, vol. 2013, Article ID 846389, 8 pages, 2013.

[19] L. Zhen, Path following with roll constraints for marine surface vessels in wave fields [Ph.D. thesis], The University of Michigan, 2009.

[20] S. Oh and J. Sun, "Path following of underactuated marine surface vessels using line-of-sight based model predictive control," Ocean Engineering, vol. 37, no. 2-3, pp. 289-295, 2010.

[21] T. I. Fossen, Marine Control Systems, Marine Cybernetics, Trondheim, Norway, 2002.

[22] F. Mazenc, K. Y. Pettersen, and H. Nijmeijer, "Global uniform asymptotic stabilization of an underactuated surface vessel," in Proceedings of the 41st IEEE Conference on Decision and Control, pp. 510-515, Las Vegas, Nev, USA, December 2002.

[23] K. Y. Wichlund, O. J. Sordalen, and O. Egeland, "Control properties of underactuated vehicles," in Proceedings of the IEEE International Conference on Robotics and Automation, vol. 2, pp. 2009-2014, Nagoya, Japan, May 1995.

[24] C. I. Byrnes and A. Isidori, "On the attitude stabilization of rigid spacecraft," Automatica, vol. 27, no. 1, pp. 87-95, 1991.

[25] K. Y. Pettersen and O. Egeland, "Exponential stabilization of an underactuated surface vessel," in Proceedings of the 35th IEEE Conference on Decision and Control, pp. 967-972, Kobe, Japan, December 1996.

[26] M. V. Kothare, V. Balakrishnan, and M. Morari, "Robust constrained model predictive control using linear matrix inequalities," Automatica, vol. 32, no. 10, pp. 1361-1379, 1996.

[27] J. R. Cloutier, C. N. D’ Souza, and C. P. Mracek, "Nonlinear regulation and nonlinear $H_{\infty}$ control via the state-dependent Riccati equation technique," in Proceedings of the International Conference on Nonlinear Problems in Aviation and Aerospace, University Press, Embry-Riddle Aeronautical University, Daytona Beach, Fla, USA, May 1996.

[28] J. R. Cloutier, "State-dependent Riccati equation techniques: an overview," in Proceedings of the American Control Conference, pp. 932-936, June 1997.

[29] S. Boyd, L. El Ghaoui, E. Feron, and V. Balakrishnan, Linear Matrix Inequalities in System and Control Theory, SIAM, Philadelphia, Pa, USA, 1994.

[30] T. Yang and H. R. Karimi, "LMI-based model predictive control for a class of constrained uncertain fuzzy Markov jump systems," Mathematical Problems in Engineering, vol. 2013, Article ID 963089, 13 pages, 2013. 
[31] H. R. Karimi, "Adaptive $H_{\infty}$ synchronization of master-slave systems with mixed time-varying delays and nonlinear perturbations: an LMI approach," International Journal of Automation and Computing, vol. 8, no. 4, pp. 381-390, 2011.

[32] H. R. Karimi, "Robust $H_{\infty}$ filter design for uncertain linear systems over network with network-induced delays and output quantization," Modeling, Identification and Control, vol. 30, no. 1, pp. 27-37, 2009.

[33] Y. Yin, Y. Liu, and H. R. Karimi, "A simplified predictive control of constrained Markov jump system with mixed uncertainties," Abstract and Applied Analysis, vol. 2014, Article ID 475808, 7 pages, 2014.

[34] S. Yin, H. Luo, and S. Ding, "Real-time implementation of faulttolerant control systems with performance optimization," IEEE Transactions on Industrial Electronics, vol. 64, no. 5, pp. 24022411, 2014.

[35] S. Yin, G. Wang, and H. Karimi, "Data-driven design of robust fault detection system for wind turbines," Mechatronics, vol. 24, no. 4, pp. 298-306, 2014.

[36] S. Yin, S. X. Ding, A. H. A. Sari, and H. Hao, "Data-driven monitoring for stochastic systems and its application on batch process," International Journal of Systems Science, vol. 44, no. 7, pp. 1366-1376, 2013.

[37] S. Yin, S. X. Ding, A. Haghani, H. Hao, and P. Zhang, "A comparison study of basic data-driven fault diagnosis and process monitoring methods on the benchmark Tennessee Eastman process," Journal of Process Control, vol. 22, no. 9, pp. 1567-1581, 2012.

[38] S. Yin, X. Yang, and H. R. Karimi, "Data-driven adaptive observer for fault diagnosis," Mathematical Problems in Engineering, vol. 2012, Article ID 832836, 21 pages, 2012. 


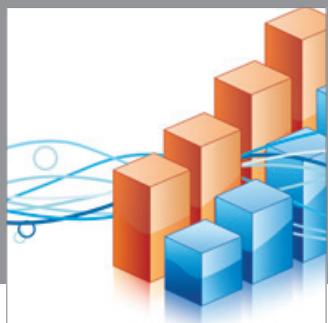

Advances in

Operations Research

mansans

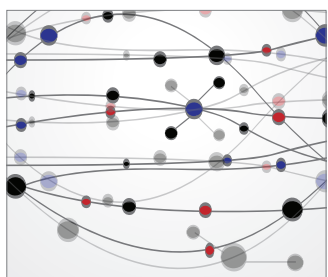

The Scientific World Journal
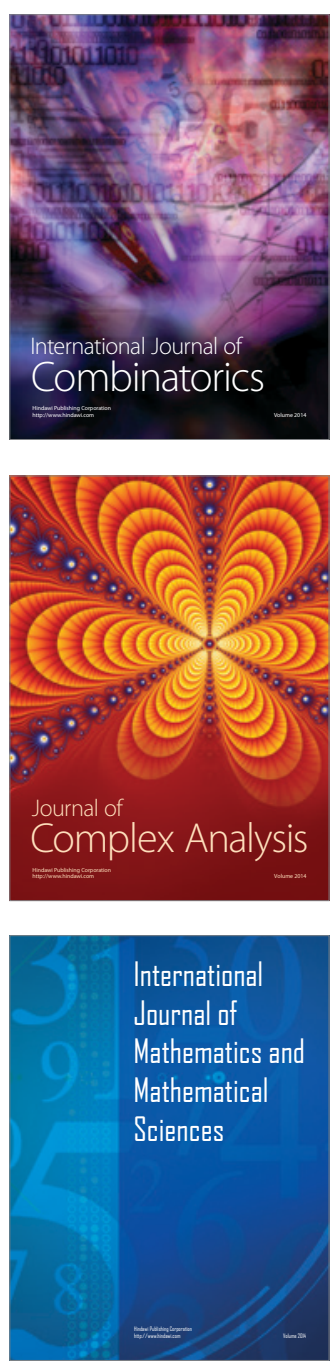
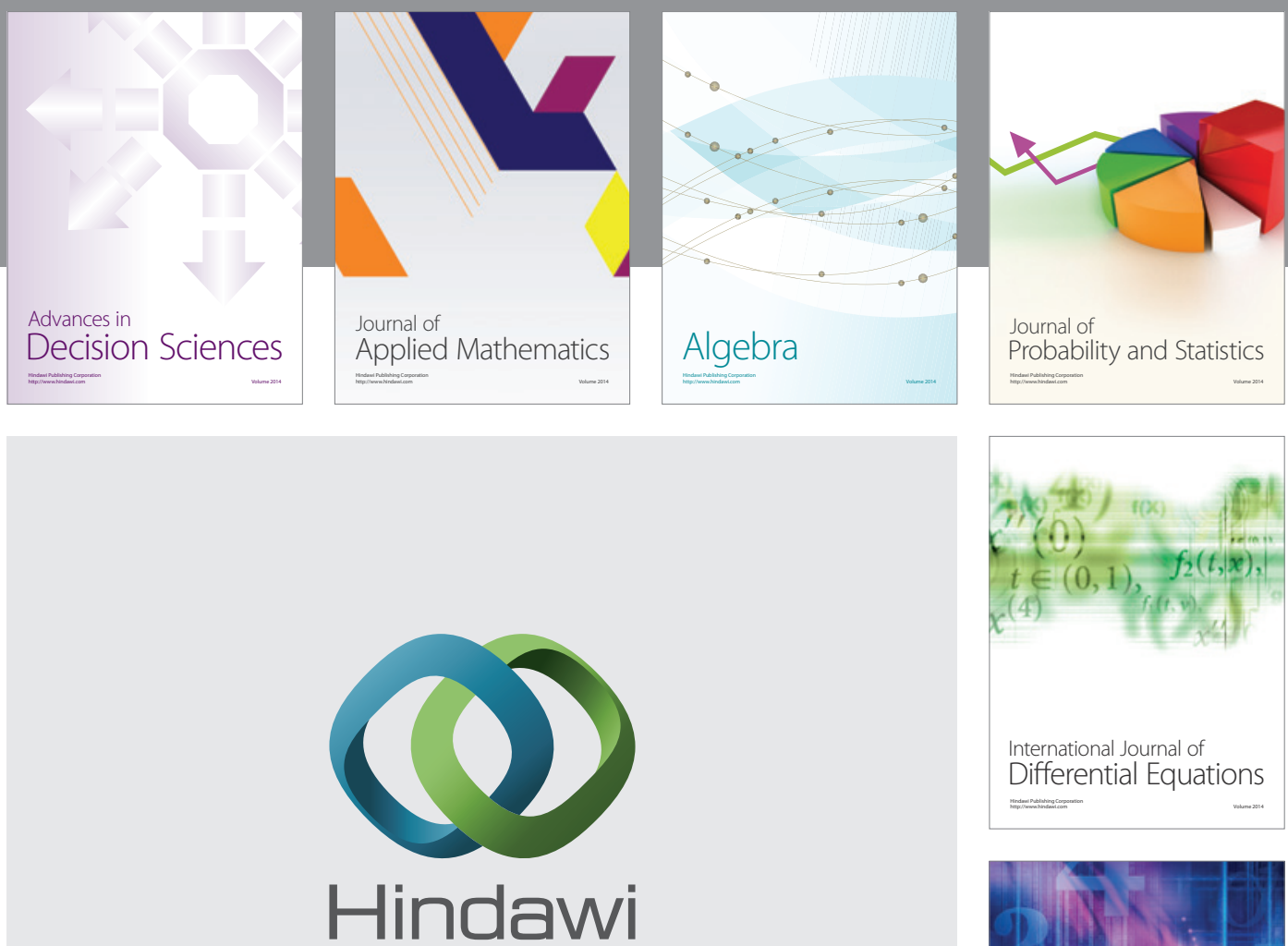

Submit your manuscripts at http://www.hindawi.com
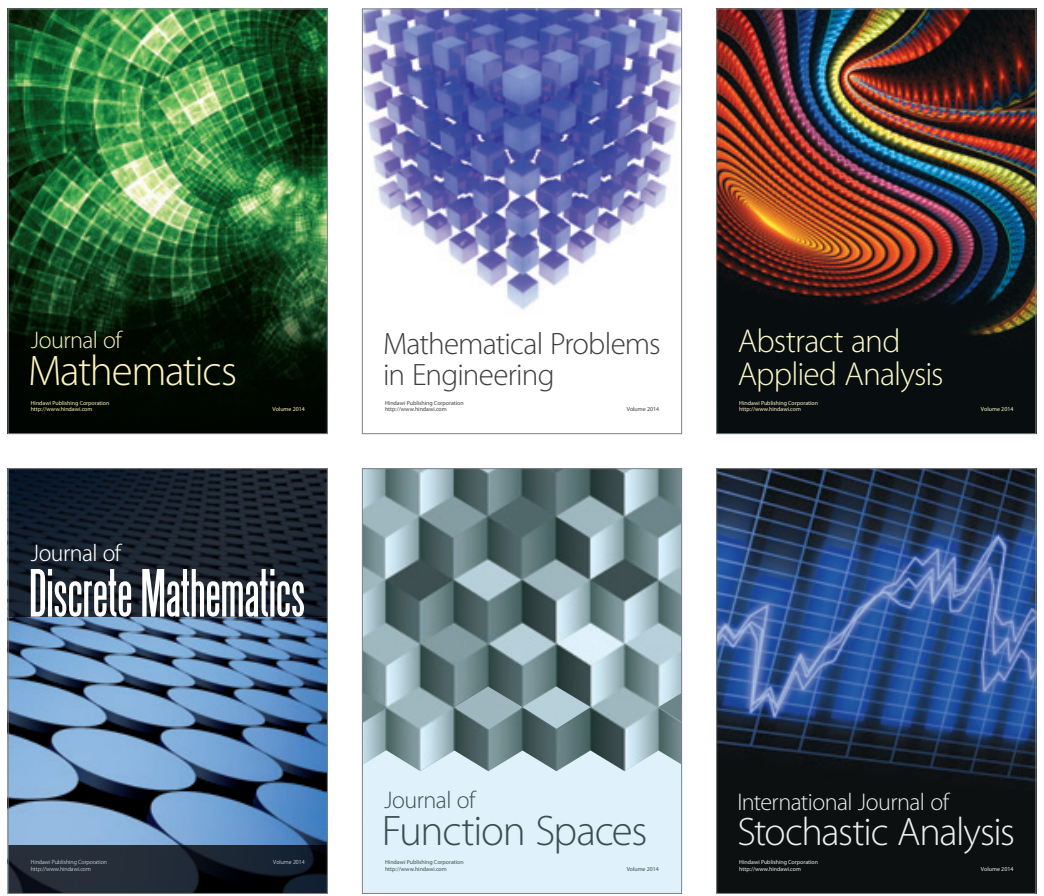

Journal of

Function Spaces

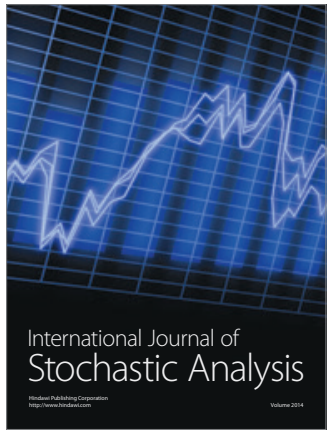

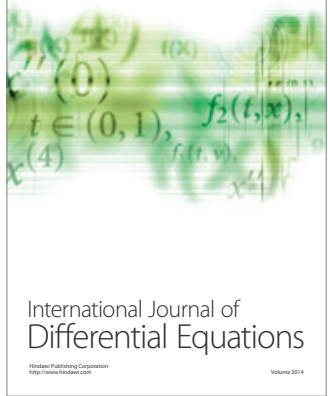
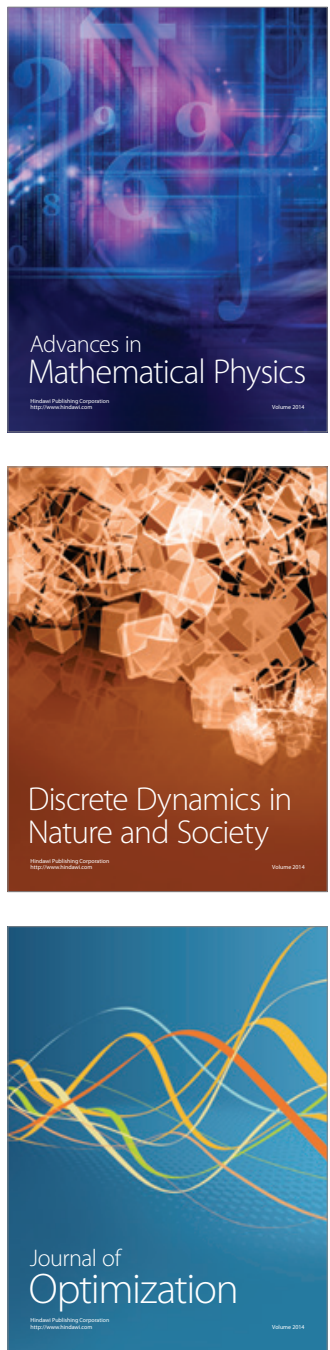